\title{
Upregulation of nuclear PA28 $\gamma$ expression in cirrhosis and hepatocellular carcinoma
}

\author{
MOTOI KONDO $^{1}$, KOHJI MORIISHI ${ }^{2}$, HIROSHI WADA $^{3}$, TAKEHIRO NODA $^{3}$, SHIGERU MARUBASHI $^{3}$, \\ KENICHI WAKASA ${ }^{4}$, YOSHIHARU MATSUURA ${ }^{2}$, YUICHIRO DOKI ${ }^{3}$, \\ MASAKI MORI ${ }^{3}$ and HIROAKI NAGANO ${ }^{3}$ \\ ${ }^{1}$ Evidence Based Medical Research Center, Osaka; ${ }^{2}$ Department of Molecular Virology, \\ Research Institute for Microbial Diseases, Osaka University, Osaka; ${ }^{3}$ Department of Gastroenterological Surgery, \\ Graduate School of Medicine, Osaka University, Osaka; ${ }^{4}$ Department of Diagnostic Pathology, \\ Graduate School of Medicine, Osaka City University, Osaka, Japan
}

Received September 16, 2011; Accepted December 2, 2011

DOI: 10.3892/etm.2011.415

\begin{abstract}
We previously reported that proteasome activator $28 \gamma(\mathrm{PA} 28 \gamma)$ is an oncogenic protein in hepatitis $\mathrm{C}$ virus (HCV) core protein transgenic mice. The aim of this study was to determine the role of PA28 $\gamma$ expression at the protein level in the development and progression of human hepatocarcinogenesis and hepatocellular carcinoma (HCC). Samples from tissues representing a wide spectrum of liver disease were analyzed, including histologically normal livers $(n=5), \mathrm{HCV}$-related chronic hepatitis $(\mathrm{CH})(\mathrm{n}=15)$ and cirrhosis $(\mathrm{n}=31)$. The level of nuclear PA28 $\gamma$ increased with the progression of liver disease from $\mathrm{CH}$ to cirrhosis. The majority of cirrhotic livers $(68 \%$; $21 / 31)$ displayed high nuclear PA28 $\gamma$ expression. However, in half of the HCCs $(50 \% ; 18 / 36)$, little or no nuclear PA28 $\gamma$ expression was observed, while the remaining $50 \%(18 / 36)$ of the cases displayed high levels of nuclear PA28 $\gamma$ expression. A clinicopathological survey demonstrated a significant correlation between nuclear PA28 $\gamma$ expression and capsular invasion in $\mathrm{HCC}(\mathrm{P}=0.026)$; a striking difference was found between nuclear PA28 $\gamma$ expression in non-tumor tissues and shorter disease-free survival $(\mathrm{P}<0.01)$. Moreover, nuclear PA28 $\gamma$ expression in non-tumor tissues correlated with the expression of molecules related to the genesis of hepatic steatosis and $\mathrm{HCC}$, such as sterol regulatory element binding protein-1c mRNA. The findings suggest the involvement of nuclear
\end{abstract}

Correspondence to: Dr Hiroaki Nagano, Department of Gastroenterological Surgery, Graduate School of Medicine, Osaka University, 2-2 Yamadaoka E-2, Suita, Osaka 565-0871, Japan

E-mail: hnagano@gesurg.med.osaka-u.ac.jp

Abbreviations: $\mathrm{CH}$, chronic hepatitis; $\mathrm{HBV}$, hepatitis B virus; $\mathrm{HCC}$, hepatocellular carcinoma; $\mathrm{HCV}$, hepatitis $\mathrm{C}$ virus; PA, proteasome activator; PBGD, porphobilinogen deaminase; RT-PCR, reverse transcription-polymerase chain reaction

Key words: proteasome activator $28 \gamma$, hepatocellular carcinoma, cirrhosis, western blotting, immunohistochemistry
PA28 $\gamma$ expression in the progression and relapse of HCC, and suggest that nuclear PA28 $\gamma$ is a potentially suitable target for the prevention and/or treatment of HCC.

\section{Introduction}

Hepatocellular carcinoma (HCC) is one of the most common malignancies worldwide, accounting for approximately $6 \%$ of all human carcinomas and 1 million deaths annually, with an estimated number of new cases of over 500,000/year (1). Clinical and experimental evidence suggests a link between infection with hepatitis $\mathrm{C}$ virus (HCV) and/or hepatitis $\mathrm{B}$ virus (HBV), chronic hepatitis $(\mathrm{CH})$ and cirrhosis, as well as the progression of HCC. Liver cirrhosis is observed in up to $90 \%$ of patients with $\mathrm{HCC}$, and $\mathrm{HCV}$ is the causative factor in $80 \%$ and HBV in $10 \%$ of cases in Japan (2-5). In the United States, almost 4 million individuals are infected with HCV each year which progresses to chronic hepatitis $\mathrm{C}$, which could potentially progress to liver cirrhosis. The results are often liver failure or HCC. Chronic hepatitis $\mathrm{C}$ is the nation's leading cause of $\mathrm{HCC}$, and according to the American Liver Foundation, is also the leading reason for liver transplantation. In Japan, HCV and/or HBV-based hepatitis and cirrhosis are also serious problems since they progress to $\mathrm{HCC}$ at a ratio of 5 to $7 \%$ per year $(4,5)$. These findings strongly suggest the existence of a link between hepatocarcinogenesis and $\mathrm{HCV} / \mathrm{HBV}$ infection and chronic liver inflammation.

Various therapies are currently in use for HCC. These include surgical resection, percutaneous ethanol injection (PEI), systemic or arterial chemotherapy using either single or combination drugs, transcatheter arterial chemoembolization (TACE), hormonal therapy and selective radiotherapy. However, the prognosis of patients with HCC remains poor, as they often develop intrahepatic and/or multicentric tumor recurrence, at a rate of $20-40 \%$ within 1 year, and $\sim 80 \%$ within 5 years of therapy even when curative treatment is applied (6-9). Liver transplantation offers the best prognosis for patients with small HCC, although its use is limited due to the scarcity of donor organs. Therefore, an effective therapeutic strategy against $\mathrm{HCC}$ is required. 
In a previous study, we reported that proteasome activator $28 \gamma$ (PA28 $\gamma$ ) directly enhances the degradation of the HCV core protein and plays a key role in the genesis of hepatic steatosis and $\mathrm{HCC}$ in $\mathrm{HCV}$ core protein transgenic mice (10). Furthermore, the above events were not observed in PA28 $\gamma$-knockout mice. The present study is an extension of our previous study and was designed to assess the utility of PA28 $\gamma$ expression as a biological marker for $\mathrm{HCV}$-related human liver disease and HCC. The findings showed the presence of high levels of nuclear PA28 $\gamma$ in multistep hepatocarcinogenesis and HCC invasion, suggesting that selective inhibitors of nuclear PA28 $\gamma$ may be useful in the prevention and/or treatment of this disease.

\section{Materials and methods}

Tissue samples. The study protocol was approved by the Human Ethics Review Committee of Osaka University, and a signed consent form was obtained from each subject for the use of tissue samples for medical research. Tissue samples were obtained from 51 patients with liver tumors, who underwent hepatectomy at the Department of Gastroenterological Surgery, Osaka University Hospital. All patients had HCV infection (28 patients) and some had HCV plus HBV infection (18 patients), but none had only HBV infection. The mean post-treatment follow-up period was $6.2 \pm 2.5$ years \pm standard deviation (SD). The excised hepatic tissue samples were examined immunohistochemically for PA28 $\gamma$ expression, including 46 paired HCCs. Non-tumor tissues were also examined, which comprised $15 \mathrm{CH}$-based livers (5 chronic active hepatitis and 10 chronic inactive hepatitis) and 31 cirrhotic livers. Prior to hepatectomy for HCC, 10 patients were treated with transarterial embolization (TAE). In these cases, histopathological examination showed complete hepatic necrosis. Histologically normal livers were also obtained from patients negative for hepatic viral infections who had liver metastasis secondary to colorectal cancer.

For immunohistochemistry, the tissue samples were fixed in $10 \%$ neutral buffered formalin, processed through graded ethanol and embedded in paraffin. The samples were frozen immediately in liquid nitrogen and stored at $-80^{\circ} \mathrm{C}$ for subsequent analysis by reverse transcription-polymerase chain reaction (RT-PCR).

Histopathological examination. Tissue sections ( $4 \mu \mathrm{m}$ thick) were deparaffinized in xylene, rehydrated and stained with hematoxylin and eosin solution. Separation of the tissues into non-tumor and tumor tissues was determined by a pathologist (K.W.) who was blinded to the clinical background. For non-tumor tissues, the presence of inflammation or cirrhotic nodules was examined. Tumor tissues were examined for the following characteristics: cell differentiation (well, moderate, poorly differentiated), number of tumors, capsular formation, septal formation, capsular invasion, portal vein tumor thrombus formation and hepatic vein invasion.

Preparation of anti-human PA28\% antibody. Chicken anti-human PA28 $\gamma$ antibody was prepared by immunization using the synthetic peptides of residues from 75 to 88 , SHDGLDGPTYKKRR, of human PA28 $\gamma$. The antibody was purified by affinity chromatography using beads conjugated with the antigen peptide.

Immunohistochemistry and evaluation of PA28 $\gamma$ immunostaining. Formalin-fixed tissues were embedded in paraffin according to the standard procedures. For immunohistochemistry, formalin-fixed tissue sections were boiled in Target Retrieval Solution (Dako, Glostrup, Denmark) and then treated with $3 \% \mathrm{H}_{2} \mathrm{O}_{2}$. The activated sections were washed twice with phosphate-buffered saline (PBS), blocked with PBS containing $5 \%$ bovine serum albumin, and incubated overnight with the purified chicken antibody to PA $28 \gamma$, followed by incubation with horseradish peroxidase-conjugated anti-chicken IgG antibody (ICN, Biomedicals, Inc., Aurora, OH, USA) as a secondary antibody. Immunoreactive antigen was visualized with 3,3'-diaminobenzidine substrate. The resulting sections were counterstained with hematoxylin. Staining of endogenous PA28 $\gamma$ with the antibody was identified in normal mouse liver sections but not in the liver sections from PA28 $\gamma$-deficient mice. Pre-immune purified antibody did not react with any other antigen in these sections under the experimental conditions.

For evaluation of PA28 $\gamma$ immunostaining, each section was scored for nuclear and cytoplasmic staining using a scale from 0 to 2 where 0 represented negative or faint staining, 1 represented moderate staining, and 2 represented strong staining. In general, the nuclei of the bile ducts faintly expressed PA28 $\gamma$ (Fig. 1a). Thus, the staining level was used as a nuclear inner control within the sample, which was designated arbitrarily as intensity level 0 . Also, slightly higher expression was designated arbitrarily as intensity level 1 and clearly higher expression was designated arbitrarily as intensity level 2 . PA28 $\gamma$ expression was very faint or undetectable in the vascular epithelia and nuclei (Fig. 1a), whereas the cytoplasm of bile duct epithelial cells and nuclei devoid of significant inflammation generally expressed faint levels of PA28 $\gamma$ (Fig. 1a). For semi-quantitative analysis, the latter level of staining was used as a cytoplasmic inner control within the sample, and designated arbitrarily as intensity level 0 . Furthermore, a slightly higher expression was designated arbitrarily as intensity level 1 whereas clearly higher expression was designated arbitrarily as intensity level 2 . PA28 $\gamma$ expression was generally heterogeneous in each sample. For assessment of nuclear and cytoplasmic PA28 $\gamma, 4$ high-power fields in each specimen were selected at random, and staining was examined under high power magnification. More than 1,000 cells were counted to determine the labeling index, which represented the percentage of immunostained cells relative to the total number of cells. The tissue samples were also categorized as positive (levels 1 and 2) and negative (level 0) for evaluation of the relationship between immunostaining and various clinicopathological factors.

Semi-quantitative RT-PCR. RNA extraction was carried out with TRIzol reagent using the single-step method, and the cDNA was generated with avian myeloblastosis virus reverse transcriptase (Promega, Madison, WI, USA), as described previously (11). Sterol regulatory element binding protein-1c (SREBP-1c) mRNA expression was analyzed semi-quantitatively using the multiplex RT-PCR method. In this assay, the 

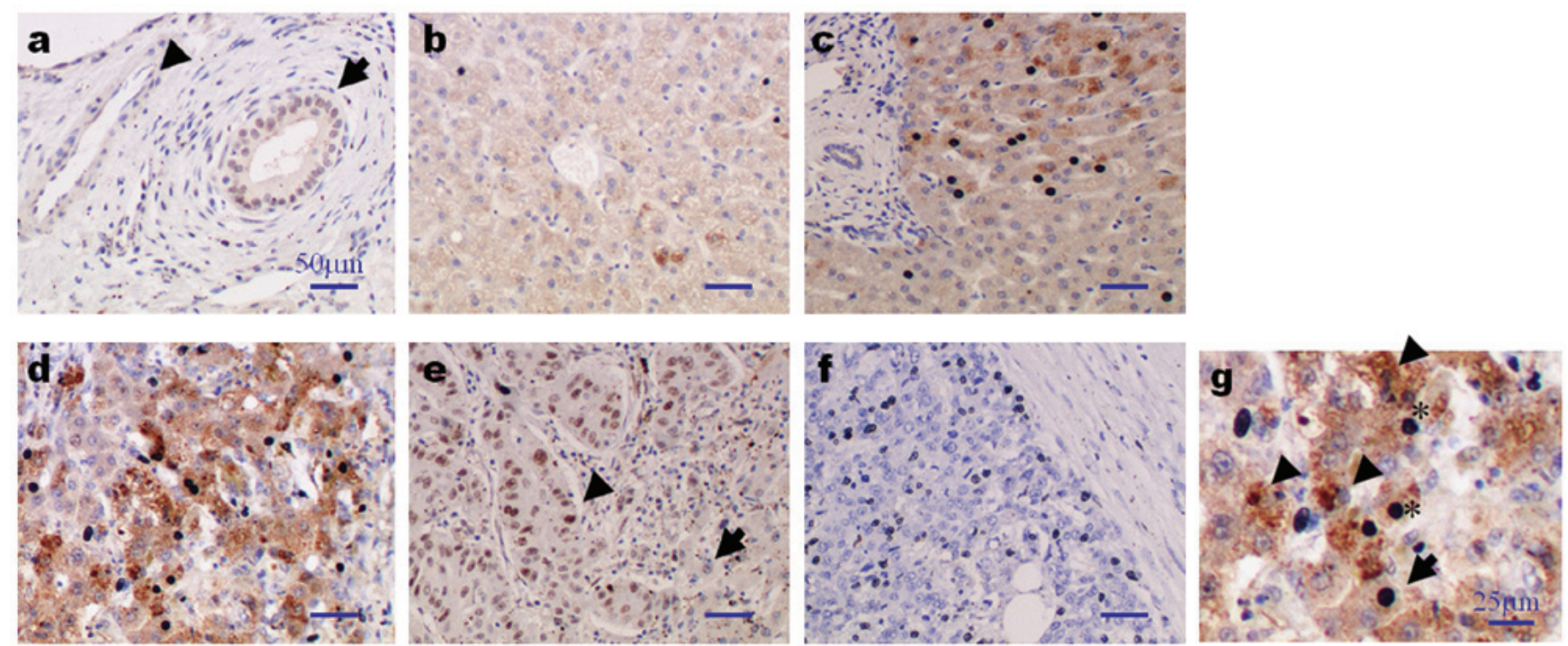

Figure 1. Immunohistochemical staining for PA28 $\gamma$. (a-f) Representative samples for bile duct (inner control), vascular epithelium and various liver pathologies; (a) bile duct (arrow), vascular epithelium (arrowhead); (b) normal liver; (c) chronic hepatitis; (d) cirrhotic liver; (e) HCC with high nuclear PA28 $\gamma$ expression (arrowhead; left side) and non-tumor liver tissue with low nuclear PA28 $\gamma$ expression (arrow; right side); (f) HCC with low expression of nuclear PA28 $\gamma$. Magnification, x200. (g) High-power view of liver section shown in (d). Note the faint staining of hepatocytes with high expression of nuclear PA28 $\gamma$ (arrow; hepatocytes, level 0 and nucleus, level 2), moderate staining of hepatocytes with high expression of nuclear PA28 $\gamma$ (asterisk; hepatocyte, level 1 and nucleus, level 2) and strong staining of hepatocytes with low expression of nuclear PA28 $\gamma$ (arrowhead; hepatocyte, level 2 and nucleus, level 0). Magnification, x400. No staining was observed when the primary antibody was substituted by non-immunized rabbit IgG or TBS (data not shown). PA28 $\gamma$, proteasome activator $28 \gamma$; HCC, hepatocellular carcinoma; IgG, immunoglobulin G; TBS, Tris-buffered saline.

housekeeping gene, porphobilinogen deaminase (PBGD), was used as the internal control. This gene is favored over $\beta$-actin or glyceraldehyde-3-phosphate dehydrogenase as a reference gene for competitive PCR amplification as the presence of pseudogenes for the latter housekeeping genes may produce false-positive signals from genomic DNA contamination $(12,13)$. In addition, in order to minimize possible inter-PCR differences, PCR was performed with SREBP-1c and PBGD primers in an identical tube, under unsaturated conditions. PCR was performed in a $25-\mu 1$ reaction mixture containing $1 \mu 1$ of the cDNA template, $1 \mathrm{X}$ Perkin-Elmer PCR buffer, $1.5 \mathrm{mM} \mathrm{MgCl} 2,0.8 \mathrm{mM}$ deoxynucleotide triphosphates, $0.8 \mu \mathrm{M}$ of each primer for SREBP-1c and $80 \mathrm{nM}$ PBGD, and 1 unit of TaqDNA polymerase (AmpliTaq Gold; Roche Molecular Systems, Inc.). The PCR primers used for the detection of SREBP-1c and PBGD cDNAs were synthesized as described previously $(14,15)$. The conditions for multiplex PCR were one cycle of denaturation at $95^{\circ} \mathrm{C}$ for $12 \mathrm{~min}$, followed by 40 cycles at $95^{\circ} \mathrm{C}$ for $1 \mathrm{~min}, 62^{\circ} \mathrm{C}$ for $1 \mathrm{~min}$ and $72^{\circ} \mathrm{C}$ for $1 \mathrm{~min}$, and a final extension at $72^{\circ} \mathrm{C}$ for $10 \mathrm{~min}$. The electrophoresed PCR products were scanned by densitometry, and the relative value of the SREBP-1c band relative to that of PBGD was calculated for each sample.

Statistical analysis. Data were expressed as the means \pm SD. The Chi-square test and Fisher's exact probability test, or the log-rank test, were used to examine the association between PA28 $\gamma$ expression and the clinicopathological parameters or prognosis. A P-value of $<0.05$ was considered to indicate a statistically significant difference. Statistical analysis was performed using the StatView-J-5.0 program (SAS Institute, Cary, NC, USA).

\section{Results}

Immunohistochemical analysis of PA28 $\gamma$. Immunohistochemical assays were performed on a series of 46 paired HCCs and their matched non-tumor tissues, and 5 normal livers. The labeling index of nuclear PA28 $\gamma$ showed a wide spectrum and increased from low in the normal livers to strong in the cirrhotic livers (Fig. 1b-d). Specifically, the nuclear PA28 $\gamma$ labeling index was generally low in the normal liver tissues, but was moderate-strong in HCV-related liver tissues. The nuclear labeling index was markedly higher in the majority of cirrhotic liver tissues. Fig. 2 summarizes the above results and the analysis of cytoplasmic expression of PA28 $\gamma$. The difference in the PA28 $\gamma$-nuclear labeling index between normal and cirrhotic livers was significant $(\mathrm{P}<0.0001)$ as was that between $\mathrm{CH}$ and cirrhosis $(\mathrm{P}<0.0001)($ Fig. 2A). Also, the difference in the proportion of the PA28 $\gamma$-cytoplasmic expression labeling index between normal and cirrhotic livers was significant $(\mathrm{P}<0.05)$ (Fig. 2B). The mean labeling indexes of nuclear PA28 $\gamma$ expression was $42 \%$ in both HCC and HCV-related livers.

To evaluate the relationship between immunohistochemical staining and various clinicopathological factors, we divided the samples into nuclear PA28 $\gamma$ high index $(\geq 42 \%)$ and low index $(<42 \%)$ groups. The labeling index was low in half of the examined HCC cases $(50 \% ; 18 / 36)$ and markedly high in the other half $(50 \% ; 18 / 36)$ (Table I). The labeling index was low in 30\% (14/46) of HCV-related cases and markedly higher in the remaining $70 \%$ (32/46) (Table II). The samples were also divided into 2 groups according to the labeling index of cytoplasmic staining. The mean PA28 $\gamma$-labeling index of the HCC and $\mathrm{HCV}$-related cases was 58 and $80 \%$, respectively. The labeling index was low in 47\% (17/36) and high in 53\% (19/36) 
A

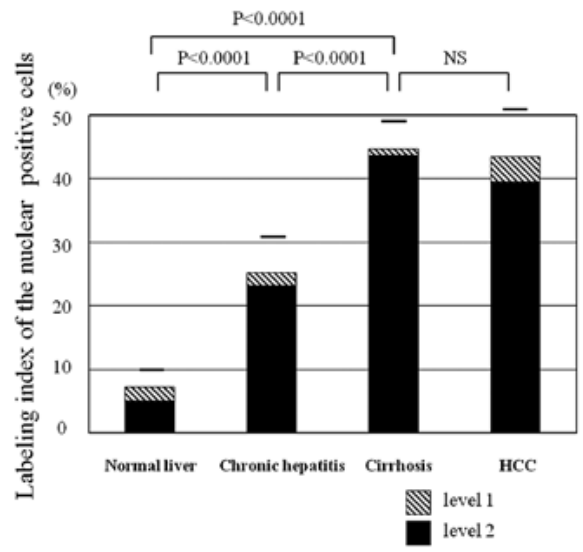

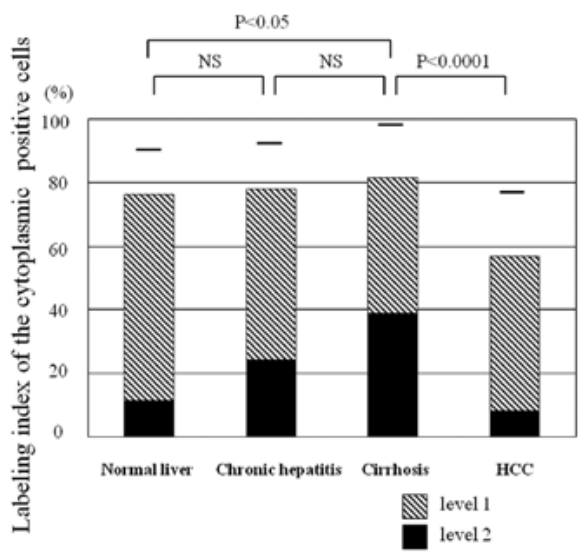

Figure 2. (A) Nuclear PA28 $\gamma$ expression in multistep hepatocarcinogenesis. The labeling index increased in a stepwise manner with the severity of liver damage and carcinogenesis. Quantitative analysis showed that 25,10 and $1 \%$ of cells of the normal liver, $\mathrm{CH}$ and cirrhosis, respectively, were moderately positive

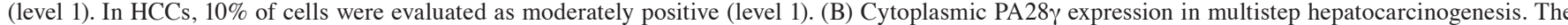
expression increased slightly in a stepwise manner. Quantitative analysis showed that 80,68 and $50 \%$ of cells of the normal liver, $\mathrm{CH}$ and cirrhosis, respectively, were moderately positive (level 1). In HCCs, $82 \%$ of cells were evaluated as moderately positive (level 1). PA28 $\gamma$, proteasome activator $28 \gamma$; $\mathrm{CH}$, chronic hepatitis; HCC, hepatocellular carcinoma. NS, not significant.

Table I. Correlation between nuclear PA28 $\gamma$ expression and various clinicopathological parameters in patients with HCC.

PA28 $\gamma$

$\mathrm{n}$

Low $(<42 \%)$

$\operatorname{High}(\geq 42 \%)$

P-value

Age (years)

$\geq 60$

15

7

8

$<60$

21

11

10

NS

Gender

Male

21

15

10

11

Female

Tumor size

$$
\leq 2 \mathrm{~cm}
$$

$>2 \mathrm{~cm}$

Histological type

Well/moderately differentiated

Poorly differentiated

Hepatic vein invasion

Yes

6

2

No

30

16

NS

Portal vein tumor thrombus

Yes

5

2

No

Number of tumors

Multiple $^{\mathrm{a}}$

Solitary

Septum formation

Yes

Capsular formation

$21 \quad 10$

11

NS

Yes

Capsular invasion

$22-12 \quad 10$
noma; NS, not significant. 
Table II. Correlation between nuclear PA28 $\gamma$ expression and various clinicopathological parameters in non-tumor liver tissues.

\begin{tabular}{|c|c|c|c|c|}
\hline & \multirow[b]{2}{*}{$\mathrm{n}$} & \multicolumn{2}{|c|}{ PA28 $\gamma$} & \multirow[b]{2}{*}{ P-value } \\
\hline & & Low $(<42 \%)$ & High $(\geq 42 \%)$ & \\
\hline \multicolumn{5}{|l|}{ Age (years) } \\
\hline$\geq 60$ & 22 & 5 & 17 & \\
\hline$<60$ & 24 & 9 & 15 & NS \\
\hline \multicolumn{5}{|l|}{ Gender } \\
\hline Male & 27 & 6 & 21 & \\
\hline Female & 19 & 8 & 11 & NS \\
\hline $\mathrm{HCV}$ & 28 & 9 & 19 & \\
\hline HBV & 0 & & & \\
\hline HCV plus HBV & 18 & 5 & 13 & NS \\
\hline \multicolumn{5}{|c|}{ Inflammatory status (HAI score) } \\
\hline Absent-mild (0-3) & 22 & 12 & 10 & \\
\hline Moderate-severe $(>4)$ & 24 & 2 & 22 & 0.0007 \\
\hline \multicolumn{5}{|c|}{ Degree of fibrosis (HAI score) } \\
\hline Absent-moderate (0-2) & 12 & 11 & 1 & \\
\hline Severe-cirrhosis $(>3)$ & 34 & 3 & 31 & $<0.0001$ \\
\hline
\end{tabular}

NS, not significant; PA28 $\gamma$, proteasome activator 28 $\gamma$; HCV, hepatitis C virus; HBV, hepatitis B virus; HAI, histological activity index.

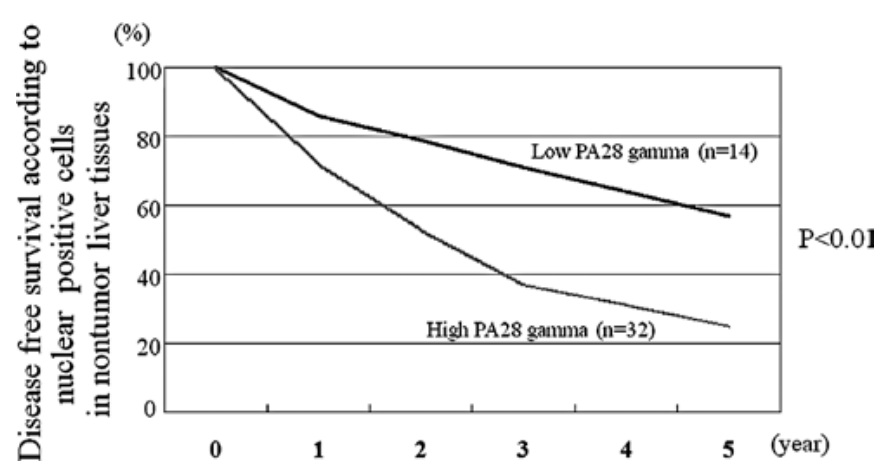

Figure 3. Disease-free survival based on nuclear PA28y expression in non-tumor tissues. The disease-free survival was significantly different between patients with high nuclear PA28 $\gamma$ expression (levels 1 and 2) and those with low nuclear PA28 $\gamma$ expression (level 0$)(\mathrm{P}<0.01)$. PA28 $\gamma$, proteasome activator $28 \gamma$.

of the HCC cases. The respective values for HCV-related cases were $28 \%$ (13/46) and $72 \%$ (33/46). All cut-off values used were according to the mean labeling index.

Correlation between nuclear PA28y expression and clinicopathological parameters. We examined the correlation between PA28 $\gamma$ nuclear expression analyzed in $36 \mathrm{HCCs}$ (10 samples with complete necrosis by TAE were excluded from this analysis) and various clinicopathological features (Table I). The cases were divided into two groups based on the labeling index of nuclear expression of PA28 $\gamma$, using a cut-off mean value of $42 \%$. There was a significant difference in PA28 $\gamma$ expression based on capsular invasion (Table I). We also analyzed the relationship between nuclear PA28 $\gamma$ expression in non-tumor tissues $(15 \mathrm{CH}$ and 31 cirrhosis) and disease-free survival, as the pathologic status of non-tumor tissues has been shown to correlate with the relapse of HCC (16-18). The disease-free survival, but not overall survival $(\mathrm{P}=0.052)$, was significantly different between high and low nuclear PA28 $\gamma$ expressors $(\mathrm{P}<0.01)$ (Fig. 3). In addition, PA28 $\gamma$ expression in non-tumor tissues correlated closely with active inflammation and fibrosis (Table II).

In univariate analysis, PA28 $\gamma$ expression in non-tumor liver tissues, portal vein tumor thrombus, inflammatory status and degree of fibrosis in the non-cancerous liver tissue were significant factors for disease-free survival. These variables were subsequently entered into multivariate analysis. The results identified nuclear PA28 $\gamma$ expression level [95\% confidence interval (CI), 1.82-3.22; $\mathrm{P}<0.01]$, portal vein tumor thrombus (95\% CI, 1.33-6.38; $\mathrm{P}=0.023)$, inflammatory status (95\% CI, 2.11-3.58; $\mathrm{P}=0.012)$ and degree of fibrosis $(95 \% \mathrm{CI}$, $1.99-7.21 ; \mathrm{P}<0.01)$ as independent factors for disease-free survival (Table III).

SREBP-1c expression. Five $\mathrm{CH}$ and five cirrhotic liver tissues were selected to analyze the correlation between nuclear PA28 $\gamma$ expression and SREBP-1c gene expression in non-tumor liver tissues. Fig. 4 shows a clear correlation between nuclear PA28 $\gamma$ expression and SREBP-1c gene expression.

\section{Discussion}

The present study shows that non-tumor liver tissues commonly express high levels of nuclear PA28 $\gamma$ protein relative to those of carcinoma tissues. These results are contradictory to those from other studies on other types of cancer, such as thyroid carcinoma; the nuclear PA28 $\gamma$ level was higher in these tumors compared to non-tumor tissues (19). While the exact reason for 
Table III. Multivariate analysis of clinicopathological factors for disease-free survival in patients with HCC.

\begin{tabular}{|c|c|c|c|c|}
\hline & $\mathrm{n}$ & Relative risk & $95 \%$ confidence interval & P-value \\
\hline \multicolumn{5}{|l|}{ PA28 $\gamma$} \\
\hline High & 32 & 2.67 & $1.82-3.22$ & $<0.01$ \\
\hline Low & 14 & & & \\
\hline \multicolumn{5}{|c|}{ Portal vein tumor thrombus } \\
\hline Yes & 5 & 2.21 & $1.33-6.38$ & 0.023 \\
\hline No & 31 & & & \\
\hline \multicolumn{5}{|c|}{ Inflammatory status (HAI score) } \\
\hline Absent-mild (0-3) & 22 & 2.59 & $2.11-3.58$ & 0.012 \\
\hline Moderate-severe (>4) & 24 & & & \\
\hline \multicolumn{5}{|c|}{ Degree of fibrosis (HAI score) } \\
\hline Absent-moderate $(0-2)$ & 12 & 2.68 & $1.99-7.21$ & $<0.01$ \\
\hline Severe-cirrhosis $(>3)$ & 34 & & & \\
\hline
\end{tabular}

HCC, hepatocellular carcinoma; PA28 $\gamma$, proteasome activator 28 $\gamma$; HAI, histological activity index.

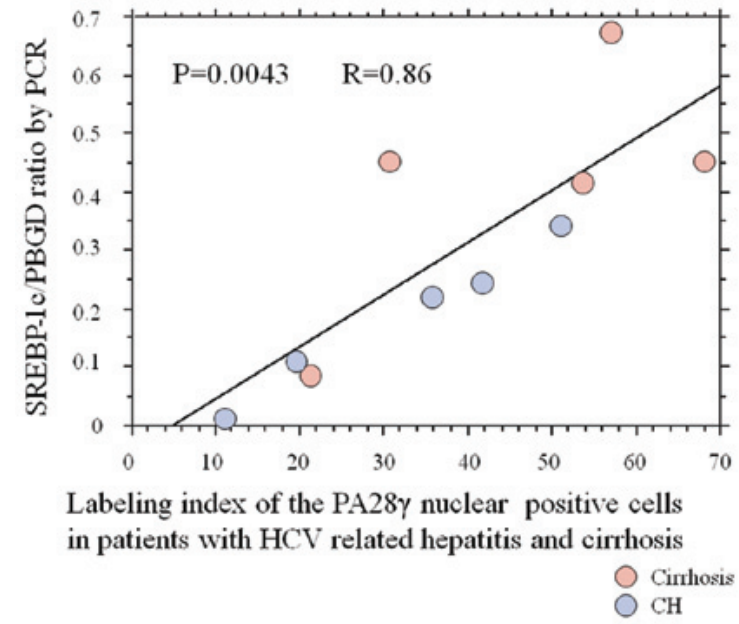

Figure 4. Linear correlation analysis of nuclear PA28 $\gamma$ expression and SREBP-1c gene expression in patients with cirrhosis and chronic hepatitis (CH) $(\mathrm{P}=0.0043)$. PA28 $\gamma$, proteasome activator $28 \gamma$; HCV, hepatitis $\mathrm{C}$ virus. SREBP-1c, sterol regulatory element binding protein-1c.

the different results is not known at present, it is likely to be related to the type of control tissue used in the present study; the non-tumor tissues were mostly not normal, consisting of $\mathrm{HCV}$-infected $\mathrm{CH}$ or cirrhotic tissues. In support of this conclusion, normal liver tissues from patients with metastatic liver tumors from patients with colorectal carcinoma who were negative for $\mathrm{HCV} / \mathrm{HBV}$ showed low expression of nuclear PA28 $\gamma$.

In non-neoplastic liver tissues, we found a wide spectrum of nuclear PA28 $\gamma$ expression from normal liver to cirrhosis. Our results also show that active inflammation with hepatitis virus induces nuclear PA28 $\gamma$ in $\mathrm{CH}$ and cirrhotic livers (Table II). This is reasonable considering the fundamental action of nuclear PA28 $\gamma$ as a mediator of inflammation. Another mechanism for the high induction of nuclear PA28 $\gamma$ in cirrhosis might be related to the degradation of the $\mathrm{HCV}$ core protein by PA28 $\gamma$ and its translocation from the cytoplasm to the nucleus, based on the results of our previous study (10). In fact, nuclear PA28 $\gamma$-expressing cells had no or faint-to-moderate cytoplasmic PA28 $\gamma$ expression (Fig. 1c and g). Furthermore, the nuclear overexpression could be due to the relatively hypoxic microenvironment in the cirrhotic liver. In this regard, we hypothesized that hypoxia might directly induce PA28 $\gamma$, which in turn enhances angiogenesis via the enhanced release of a battery of angiogenic growth factors, such as vascular endothelial growth factor (VEGF). Since the VEGF level is increased in cirrhosis (20), it is possible that nuclear PA28 $\gamma$ may improve the ischemic/hypoxic microenvironment in the cirrhotic liver through upregulation of angiogenesis. Although cirrhotic nodules occasionally show p53 mutation and increased telomerase activity $(21,22)$, cirrhosis is not considered a premalignant lesion. However, it is apparent from a number of etiological studies that cirrhosis is a strong risk factor for HCC. In this context, nuclear PA28 $\gamma$ expression in cirrhosis might be a prerequisite for the genesis of premalignant dysplastic nodules or early cancer.

From a clinical point of view, it is interesting to note the correlation between high nuclear PA28 $\gamma$ expression in non-tumor tissues and the relapse of HCC. The prognosis of HCC is generally unfavorable. Although primary tumors are curatively resected, 50-60\% of patients develop relapse within 5 years. This is due to either a newly established tumor from the remnant liver, a process termed multicentric carcinogenesis, or recurrence of the original tumor. One possible mechanism for a link between nuclear PA28 $\gamma$ and disease relapse is that high expression of PA28 $\gamma$ in the remnant liver may contribute to carcinogenesis. Nuclear PA28 $\gamma$ expression highly correlated with the presence of active inflammation $(\mathrm{P}<0.0001)$. Furthermore, active inflammation in non-tumor tissues has been reported to be associated with relapse of HCC $(17,23,24)$.

In the present study, a clinicopathological survey demonstrated a significant correlation between nuclear PA28 $\gamma$ protein expression and capsular invasion of the cancer tissue. This 
finding is in agreement with a recent study that showed increased expression of PA28 $\gamma$ protein during cancer progression and its correlation with PCNA labeling index (19). Thus, the results suggest the possible involvement of PA28 $\gamma$ in HCC progression. Further studies of larger population samples are required to confirm the clinical significance of nuclear PA28 $\gamma$ in HCC. This is particularly important, as the overall survival of patients with high nuclear PA28 $\gamma$ expression was worse than that of those with low expression level ( $\mathrm{P}=0.052)$ (data not shown).

Also in our series, the labeling index of cytoplasmic expression of PA28 $\gamma$ significantly increased from normal liver to cirrhotic liver (Fig. 2b). Further extended studies are required to determine the importance of cytoplasmic expression of PA28 $\gamma$ in HCC and HCV-related liver.

In conclusion, the present study demonstrates a close correlation between nuclear PA28 $\gamma$ expression in liver tissue and the development and progression of $\mathrm{HCC}$, as well as its possible involvement in HCC relapse. Further studies are required to examine the therapeutic benefits of the suppression of nuclear $\mathrm{PA} 28 \gamma$ expression in $\mathrm{HCV}$-related $\mathrm{CH}$, cirrhosis or $\mathrm{HCC}$.

\section{References}

1. Montalto G, Cervello M, Giannitrapani L, Dantona F, Terranova A and Castagnetta LA: Epidemiology, risk factors, and natural history of hepatocellular carcinoma. Ann NY Acad Sci 963: 13-20, 2002.

2. Okuda K: Hepatocellular carcinoma: Recent progress. Hepatology 15: 948-963, 1992.

3. Kew MC and Popper H: Relationship between hepatocellular carcinoma and cirrhosis. Semin Liver Dis 4: 136-146, 1984.

4. Ikeda K, Saitoh S, Koida I, Arase Y, Tsubota A, Chayama K, Kumada $\mathrm{H}$ and Kawanishi M. A multivariate analysis of risk factors for hepatocellular carcinogenesis: A prospective observation of 795 patients with viral and alcoholic cirrhosis. Hepatology 18: 47-53, 1993

5. Shiratori Y, Shiina S, Imamura M, et al: Characteristic difference of hepatocellular carcinoma between hepatitis B- and C-viral infection in Japan. Hepatology 22: 1027-1033, 1995.

6. Nagasue N, Uchida M, Makino Y, et al: Incidence and factors associated with intrahepatic recurrence following resection of hepatocellular carcinoma. Gastroenterology 105: 488-494, 1993.

7. Ikeda K, Saitoh S, Tsubota A, Arase Y, Chayama K, Kumada H, Watanabe $\mathrm{G}$ and Tsurumaru M: Risk factors for tumor recurrence and prognosis after curative resection of hepatocellular carcinoma. Cancer 71: 19-25, 1993.

8. Shimada M, Takenaka K, Gion T, et al: Prognosis of recurrent hepatocellular carcinoma: a 10-year surgical experience in Japan. Gastroenterology 111: 720-726, 1996.

9. Kumada T, Nakano S, Takeda I, et al: Patterns of recurrence after initial treatment in patients with small hepatocellular carcinoma. Hepatology 25: 87-92, 1997.
10. Moriishi K, Mochizuki R, Moriya K, et al: Critical role of PA28gamma in hepatitis C virus-associated steatogenesis and hepatocarcinogenesis. Proc Natl Acad Sci USA 104: 1661-1666, 2007.

11. Myers J, Mehta P, Hunter AW, Bernstein SA and Erickson PA. Automated double-label immunohistochemistry. J Surg Pathol 1: 105-113, 1995.

12. Chretien S, Dubart A, Beaupain D, et al: Alternative transcription and splicing of the human porphobilinogen deaminase gene result either in tissue-specific or in housekeeping expression. Proc Natl Acad Sci USA 85: 6-10, 1988.

13. Nagel S, Schmidt M, Thiede C, Huhn D and Neubauer A: Quantification of Bcr-Abl transcripts in chronic myelogenous leukemia (CML) using standardized, internally controlled, competitive differential PCR (CD-PCR). Nucleic Acids Res 24: 4102-4103, 1996.

14. Kim KH, Hong SP, Kim K, Park MJ, Kim KJ and Cheong J: HCV core protein induces hepatic lipid accumulation by activating SREBP1 and PPARgamma. Biochem Biophys Res Commun 355: 883-888, 2007.

15. Finke J, Fritzen R, Ternes P, Lange W and Dolken G: An improved strategy and a useful housekeeping gene for RNA analysis from formalin-fixed, paraffin-embedded tissues by PCR. Biotechniques 14: 448-453, 1993.

16. Sasaki Y, Imaoka S, Fujita M, et al: Regional therapy in the management of intrahepatic recurrence after surgery for hepatoma. Ann Surg. 206: 40-47, 2007.

17. Ko S, Nakajima Y, Kanehiro H, et al: Significant influence of accompanying chronic hepatitis status on recurrence of hepatocellular carcinoma after hepatectomy. Result of multivariate analysis. Ann Surg 224: 591-595, 1996.

18. Sasaki Y, Imaoka S, Masutani S, Ohashi I, Ishikawa O, Koyama H and Iwanaga T: Influence of coexisting cirrhosis on long-term prognosis after surgery in patients with hepatocellular carcinoma. Surgery 112: 515-21, 1992.

19. Okamura T, Taniguchi S, Ohkura T, et al: Abnormally high expression of proteasome activator-gamma in thyroid neoplasm. J Clin Endocrinol Metab 88: 1374-1383, 2003.

20. El-Assal ON, Yamanoi A, Soda Y, et al: Clinical significance of microvessel density and vascular endothelial growth factor expression in hepatocellular carcinoma and surrounding liver: possible involvement of vascular endothelial growth factor in the angiogenesis of cirrhotic liver. Hepatology 27: 1554-1562, 1998.

21. Raedle J, Oremek G, Truschnowitsch M, Lorenz M, Roth WK, Caspary WF and Zeuzem S: Clinical evaluation of autoantibodies to p53 protein in patients with chronic liver disease and hepatocellular carcinoma. Eur J Cancer 34: 1198-1203, 1998.

22. Kishimoto Y, Shiota G, Kamisaki Y, et al: Loss of the tumor suppressor p53 gene at the liver cirrhosis stage in Japanese patients with hepatocellular carcinoma. Oncology 54: 304-310, 1997.

23. Tarao K, Takemiya S, Tamai S, et al: Relationship between the recurrence of hepatocellular carcinoma (HCC) and serum alanine aminotransferase levels in hepatectomized patients with hepatitis $\mathrm{C}$ virus-associated cirrhosis and HCC. Cancer 79: 688-694, 1997.

24. Ko S, Nakajima Y, Kanehiro H, et al: Influence of associated viral hepatitis status on recurrence of hepatocellular carcinoma after hepatectomy. World J Surg 21: 1082-1086, 1996. 